\title{
Photorefractive acoustoelectro-optic correlator
}

\author{
Hyuk Lee \\ Department of Electrical Engineering and Computer Science, Polytechnic University, 333 Jay Street, Brooklyn, New York 11201
}

Demetri Psaltis

Department of Electrical Engineering, California Institute of Technology, Pasadena, California 91125

Received January 21, 1987; accepted March 25, 1987

\begin{abstract}
An optical correlator based on acoustoelectro-optic interaction is proposed. The device is experimentally demonstrated with a $\mathrm{LiNbO}_{3}$ crystal in which acoustic waves are launched by a piezoelectric transducer and electric fields are established through the photorefractive effect.
\end{abstract}

The homogeneous acoustoelectro-optic (AEO) effect is an indirect interaction of light waves with a homogeneous applied electric field and an acoustic wave that is launched in the same dielectric medium. The homogeneous AEO effect was previously analyzed and used to implement a new optical modulator, a deflector, and a tunable filter. ${ }^{1-4}$ Here we discuss the inhomogeneous AEO interaction and describe a correlator based on this effect.

The inhomogeneous AEO interaction takes place when light propagates through a crystal in which spatially varying electric fields are applied and acoustic waves propagate as well. As in the homogeneous case, the polarization field induced in the crystal is the sum of two components that are due to the linear electrooptic and acousto-optic effects. The spatial variation of the applied electric field makes the analysis of the inhomogeneous AEO effect more difficult, however. In this Letter we consider the simplest case, in which both the electric and the acoustic signals consist of a single spatial frequency, i.e., they are both sinusoidal gratings. In this case the AEO effect can be treated as double diffraction by using relatively simple coupledmode analysis. The results of the simple harmonic analysis can in principle be used to predict the diffraction for arbitrary spatial distributions of the acoustic and electric fields by Fourier transforming the two fields and treating each pair of frequency components separately. This approach will be valid if both the applied electric and acoustic signals are on a spatial carrier and the light wave is approximately phase matched to both gratings. This condition is valid for the correlator implementation that is discussed in this Letter. Specifically, we use a $\mathrm{LiNbO}_{3}$ crystal in which acoustic fields are launched by a piezoelectric transducer attached to the crystal and the inhomogeneous electric field distribution is generated by recording a hologram in the same crystal using the photorefractive effect. ${ }^{5}$ We describe a simple device implemented in this geometry that calculates the correlation ${ }^{6}$ between the electrical signal applied to the piezoelectric transducer and the pattern used to record the hologram.
The wave-matching diagram and the corresponding device geometry of one possible configuration of the photorefractive AEO correlator are shown in Figs. 1(a) and $1(\mathrm{~b})$, respectively. A photorefractive grating is optically formed with its wave vector pointing in the $y$ direction, and a single-frequency acoustic wave is launched in the $x$ direction. A plane wave with amplitude $F_{1}$ is made incident at an angle that satisfies the Bragg condition for isotropic diffraction by the photorefractive grating, resulting in the generation of an intermediate wave with amplitude $F_{2}$ that is Bragg matched for anisotropic diffraction by the acoustic wave. Thus the intermediate wave is rediffracted to produce the final doubly diffracted wave, whose amplitude $F_{3}$ is proportional to the product of the amplitudes of the two gratings. If the diffraction efficiencies of the photorefractive and acoustic gratings are small, then the coupled-mode equations of the three optical modes are

$$
\begin{aligned}
& \mathrm{d} F_{2} / \mathrm{d} r=j \mu_{12} F_{1}, \\
& \mathrm{~d} F_{3} / \mathrm{d} r=j \mu_{23} F_{2},
\end{aligned}
$$

where $r$ is the distance along which light propagates. $\mu_{12}$ and $\mu_{23}$ are the coupling coefficients of the photorefractive and acoustic gratings, respectively, and they are proportional to the amplitudes of the corresponding phase gratings. For small diffraction efficiencies we can assume that $F_{1}$ is constant, and we assume the following initial conditions:

$$
F_{1}(0)=F_{0}, \quad F_{2}(0)=0, \quad F_{3}(0)=0 .
$$

If we define diffraction efficiencies $\eta_{12}$ and $\eta_{23}$ as $\eta_{12}=$ $\left(\left|\mu_{12} L\right|\right)^{2}$ and $\eta_{23}=\left(\left|\mu_{23} L\right|\right)^{2}$, where $L$ is the interaction length, we obtain the following equations for the light intensities from Eqs. (1)-(3):

$$
\begin{aligned}
& I_{2}=\eta_{12}\left|F_{0}\right|^{2}, \\
& I_{3}=(1 / 4) \eta_{12} \eta_{23}\left|F_{0}\right|^{2} .
\end{aligned}
$$

The previous analysis is valid for a perfect phasematching condition, which can be satisfied only if both 


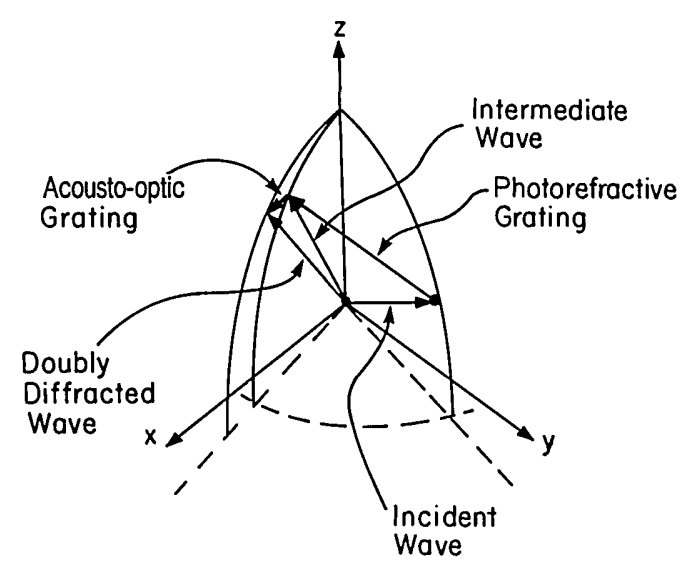

(a)

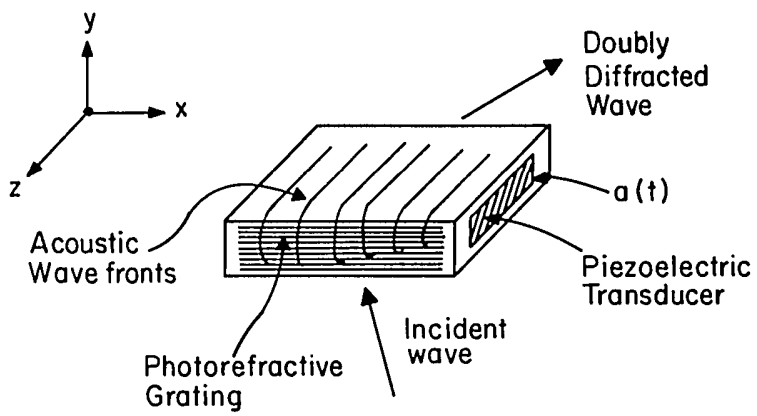

(b)

Fig. 1. (a) Wave-vector diagram of the photorefractive AEO correlator. (b) Configuration of the correlator.

the acoustic and the electric gratings consist of a single carrier frequency. This is the case when the signal $a(t)$ that drives the piezoelectric transducer is a sinusoid and the photorefractive grating is produced by interfering two plane waves.

Now suppose that the signal $a(t)$ has the form $f(t) \cos \left(\omega_{1} t\right)$, where $\omega_{1}$ is the carrier frequency and $f(t)$ is a signal that modulates its amplitude. Further suppose that the photorefractive grating that is recorded in the crystal has a spatial variation proportional to $h(x) \cos \left(\omega_{2} y\right)$, where $\omega_{2}$ is the carrier frequency of the photorefractive grating. The amplitude of the doubly diffracted light will be approximately proportional to

$$
F_{3} \sim f(t+x / v) h(x)
$$

if the following conditions are satisfied:

(1) The maximum spatial-frequency component of the signal $h(x)$ is small enough that the diffraction of the incident light into the intermediate wave is not Bragg detuned.

(2) The sum of the maximum spatial-frequency components of the signals $h(x)$ and $f(+x / v)$ is small enough that the diffraction of the intermediate wave to the doubly diffracted wave is not Bragg detuned.

In the configuration that we describe here the first condition is easily satisfied because the modulation $h(x)$ varies in a direction perpendicular to the direc- tion in which the center frequency grating is formed. It has been shown ${ }^{7}$ that this geometry maximizes the interaction bandwidth. The second condition is similar to the requirement encountered in a conventional acousto-optic deflector, except that in this case we have two separate sources of phase mismatch. If we assume that the spatial bandwidths of the signals $h(x)$ and $f(+x / v)$ are equal, then the bandwidth of the photorefractive AEO correlator is approximately equal to

$$
\Delta f=\left({ }^{1} / 2\right)\left\{\left(n_{o} \Lambda^{2} / \lambda L\right) /\left[1+\Lambda n_{e}(\vartheta) \sin \vartheta / \lambda\right]\right\} f_{c},
$$

where $\lambda$ is the optical wavelength, $\Lambda$ is the wavelength of the acoustic center frequency, $n_{o}$ is the ordinary index of refraction, $n_{e}$ is the extraordinary index of refraction, $f_{c}$ is the acoustic center frquency, and $\vartheta$ is the angle between the $z$ direction and the intermediate optical wave. Equation (7) is one half of the bandwidth of a conventional acousto-optic deflector. If

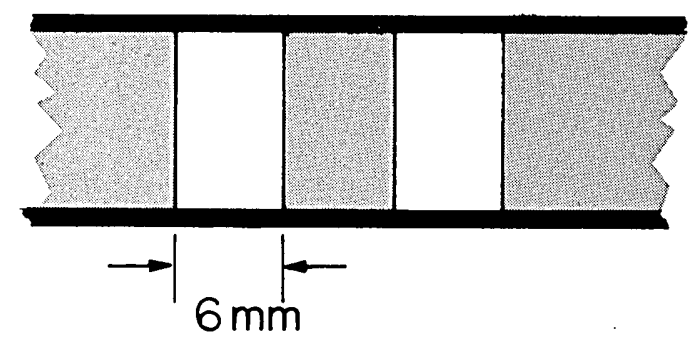

Fig. 2. Input pattern for the autocorrelation experiment.

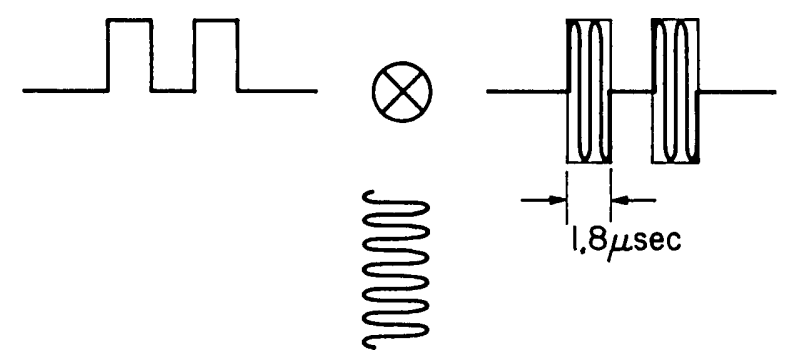

(a)

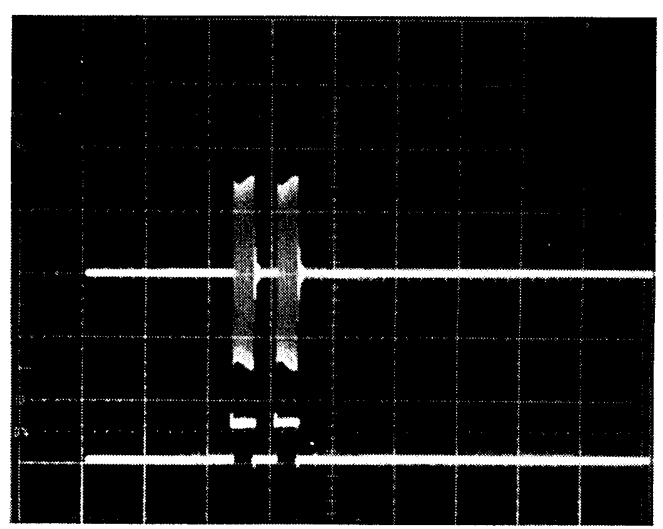

(b)

Fig. 3. (a) Modulation of the input electrical signal to the acousto-optic device. (b) Oscilloscope traces of the modulated electrical signal (top) and the two windows corresponding to the input pattern (bottom). 


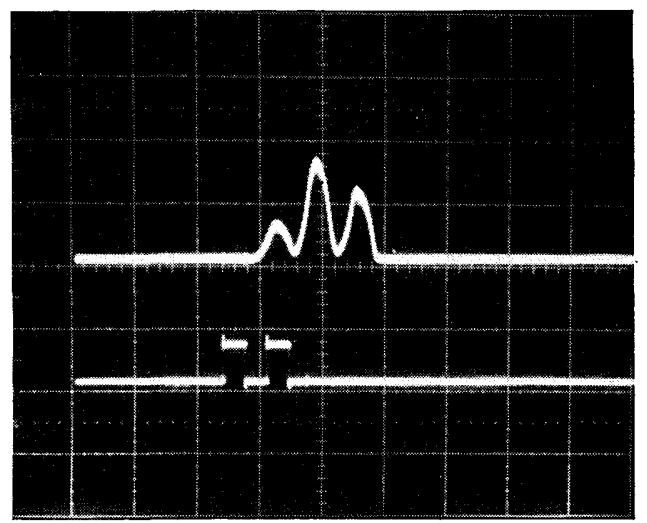

Fig. 4. Oscilloscope traces of the incoherent autocorrelation (top) and the two windows (bottom).

these two conditions are satisfied, then a correlator can be built if we place a spherical lens with focal length $f$ after the crystal. The amplitude of the doubly diffracted light at the local plane becomes

$$
\int h(x) f(t+x / v) \exp \left[j\left(x x_{1} / \lambda f\right)\right] \mathrm{d} x,
$$

where $\lambda$ is the wavelength and $x_{1}$ is the spatial coordinate at the local plane. If we use a pinhole detector located at $x_{1}=0$, we obtain the squared magnitude of the coherent correlation between $h(x)$ and $f(t+x / v)$ :

$$
I_{\text {coh }}(t)=\left|\int h(x) f(t+x / v) \mathrm{d} x\right|^{2} .
$$

If instead we collect all the light at the back focal plane, we obtain the incoherent correlation

$$
I_{\text {inc }}(t)=\int|h(x)|^{2}|f(t+x / v)|^{2} \mathrm{~d} x .
$$

To demonstrate the correlation by the above method, an autocorrelation experiment was performed with an AEO device in the configuration of Fig. 1 fabricated with $\mathrm{LiNbO}_{3}$. The acoustic center frequency of the device was $20 \mathrm{MHz}$ and its bandwidth approximately 7 $\mathrm{MHz}$. The acoustic wave propagating in the $x$ direction was a shear wave leading to anisotropic acoustooptic diffraction through the $p_{41}$ elasto-optic coefficient. The interaction length of the device was $1 \mathrm{~cm}$, and the light propagated in the near $z$ direction with an angle $1.8 \mathrm{deg}$ from the $z$ direction. The electric field distribution was photorefractively recorded in the $\mathrm{LiNbO}_{3}$ crystal as follows. First, the acoustooptic device was driven by the acoustic center frequency, and it was oriented to maximize the acousto-optic diffraction efficiency. Then two collimated Ar laser beams ( $488 \mathrm{~nm})$, satisfying the Bragg condition for the acoustic center frequency, were made incident at an angle such that their interference resulted in the formation of a grating in the $y$ direction. Two slits (Fig. 2 ) oriented in the $x$ direction were placed in front of the $\mathrm{LiNbO}_{3}$ crystal to write the two-slit pattern as an amplitude modulation of the high-frequency interference grating inside the crystal. The angle between the two interfering beams was $2.6 \mathrm{deg}$, and the diffraction efficiency was $0.6 \%$. In the experiment the intensity ratio of the two windows of the pattern in Fig. 2 was set to approximately 1 to 2 . An electrical signal was generated that exactly matched the pattern written inside the crystal when the signal was modulated by the 20 $\mathrm{MHz}$ acoustic center frequency and fed into the piezoelectric transducer. The electrical signals are shown in Fig. 3. Following these procedures we used one of the two laser beams, which were used for the formation of the interference grating, as the incident light wave in the presence of the recorded photorefractive pattern and the modulated acoustic wave. To obtain the correlation, a spherical lens collected all the doubly diffracted light at the focal plane. An oscilloscope trace of the detected light intensity is shown in Fig. 4. This is the incoherent autocorrelation of the pattern shown in Fig. 2. The ratio of the peaks is 1:3:2, as expected from the intensity ratio of the two windows of the pattern written inside the crystal.

\section{References}

1. D. Psaltis, H. Lee, and G. Sirat, Appl. Phys. Lett. 46, 215 (1985).

2. H. Lee, Appl. Phys. Lett. 49, 24 (1896).

3. P. Das, A. V. Scholtz, A. J. Urillo, D. M. Litynski, and D. Shklarsky, Appl. Phys. Lett. 49, 1016 (1986).

4. J. J. Wiczer and H. Merkelo, Appl. Phys. Lett. 30, 439 (1976).

5. F. S. Chen, J. T. LaMacchia, and D. B. Fraser, Appl. Phys. Lett. 13, 223 (1968).

6. W. T. Rhodes, Proc. IEEE, 69, 65 (1981).

7. A. Marrakchi, A. R. Tanguay, Jr., J. Yu, and D. Psaltis, Opt. Eng. 24, 124 (1985). 